\title{
A Novel Web Application for Image Fusion
}

\author{
V. Aslantas, R. Kurban, A.N. Toprak, E. Bendes \\ Erciyes University, Computer Engineering Department \\ Kayseri, TURKEY \\ \{aslantas, rkurban, antoprak, ebendes\}@erciyes.edu.tr
}

\begin{abstract}
A novel interactive web application for multi focus and multi sensor image fusion is presented in this paper. Basic averaging, Laplacian pyramid, discrete wavelet transform, block-based fusion, spatial domain multi focus image fusion and optimized region based image fusion methods are also included. Users can explore several image fusion methods and compare these methods easily and efficiently by making use of the web application.
\end{abstract}

\section{Keywords_-image fusion; multi-focus; multi-sensor; web based application}

\section{INTRODUCTION}

Recently, considering the wide use of imaging technologies, the importance and popularity of image fusion methods has increased. Image fusion has become a significant sub-area of image processing and computer vision [1]. Image fusion is an image processing technique that produces a single synthetic image that contains more complementary information than each of the images of a scene or object in which the images are taken with either more than one sensor or a single sensor with different optical parameters [2].

Fusion of the images obtained by a single sensor with different optical parameters is named as multi-focus image fusion. Optical imaging cameras are seriously affected by the problem of the finite depth of field which means the objects at different distances from the sensor cannot be focused at the same time. As a result, some objects appear in focus (sharp) others defocused (blurred). Multi-focus image fusion is aims combining the individual images with different focuses of the same scene or object to gather an everywhere-in focus image [3].

The other image fusion field that included in the proposed image fusion application is multi-sensor image fusion. The aim of the multi-sensor image fusion is obtaining a single composite image by combining the images taken from different sensors [4]. Through this way, obtained fused image is more useful than each of the source images in many areas such as medical imaging, industrial imaging and military applications [5].

Image fusion concepts can be comprehended better by visualizing the fusion processes and results. Thus, image fusion applications should be implemented by visual and interactive and easy accessible web interfaces [6]. Moreover, users have a tendency to explore the theory and applications of the methods by evaluating them with numerous source images. Recently, some interactive applications that includes commercial software packages, non-commercial toolkits [7, 8] and online HTML based materials, are used to overcome these limitations [9]. However, the non-commercial toolkits have to be downloaded before using. Furthermore, some of them are often require a commercial or academic license. Despite the fact that image fusion related web sites are easy accessible, they are static and do not contain interactive interfaces.

In this paper, a web based image fusion application is presented for multi-focus and multi-sensor image fusion that includes some well-known methods. The main advantages of the proposed application are given as follows:

- Easy accessibility,

- Visualization of the image fusion methods that allow users to comprehend the fusion concepts,

- Comparing the methods by means of fusion performance and robustness,

- Evaluating the methods with differing source images and parameter values.

Furthermore, the users can reach the proposed web based application from everywhere without installing any preliminary other software packages.

The rest of the paper is organized as follows: in the second section, the fusion methods and quality metrics that can be used on web application are explained; in third section, the interactive web based image fusion application is presented; finally, in the last section, conclusions are discussed.

\section{IMAGE FUSION METHODS AND QUALITY METRICS}

\section{A. Image fusion methods}

In this section, image fusion methods available on the web based application are described. 
The Laplacian pyramid (LP) consists of filtered and subsampled versions of the original image. The lowest level of the pyramid is the original image. To construct a level of LP, procedures of blurring, reducing image size, interpolating and differencing are employed successively on the previous pyramid level [10]. These procedures are repeated until the envisioned level of the pyramid is reached.

Discrete wavelet transform (DWT) is one of the wellknown multi scale decomposition based image fusion methods. To construct the first level of DWT, low-pass and the high-pass filtering followed by down-sampling operations are applied on each row and column of the original image, respectively. By this way, the four DWT sub bands are obtained for the first level of the decomposition. These procedures are recursively repeated on the approximation sub band that contains the horizontal and vertical lower frequencies, until the preferred decomposition level is reached [11].

In the LP and DWT based fusion, first of all, the source images are transformed to a pyramid. Then, the fused coefficient is obtained by selecting maximum coefficients from each corresponding position on the pyramids of the source images. Finally, the fused image is reconstructed by performing an inverse transform.

In block based image fusion (BBIF), first, the source images are divided into equal-sized blocks without spaces or overlaps between the adjacent blocks. Then, for each corresponding block pair, a focus measure is applied to calculate sharpness. The fused image is constructed by copying blocks with the higher sharpness value [12].

Since there is no evidence of how sensitive the fused image to several different values of the block size, a suitable block size needs to be decided. To overcome this problem, the block size is optimized in spatial domain multi focus image fusion (SDMIF) [13].

The optimized region based multi sensor image fusion (ORMSIF) method is based on DWT [14]. Rather than choosing maximum coefficient of DWT, a weighted average of coefficient is utilized. Instead of establishing fusion rules for all the coefficients of the sub bands, the weights are fixed by an optimization algorithm for predetermined regions.

\section{B. Quality metrics}

A sharp image contains more dispersed pixel values than the blurred one. Hence, variance of the image is a criterion for measuring the quality of image. For an $M \times N$ size of fused image $(f)$, the variance is computed as:

$$
V A R=\frac{1}{M \times N} \sum_{i} \sum_{j}(f(i, j)-\bar{f})^{2}
$$

where $\bar{f}$ is the average grey level over the image region:

$$
\bar{f}=\frac{1}{M \times N} \sum_{i} \sum_{j} f(i, j)
$$

Mutual information $(M I)$ measures the shared information between reference and fused image by using Kullback-Leibler measure as follows [15]:

$$
M I_{R F}=\sum_{i, j} P_{R F}(i, j) \log \frac{P_{R F}(i, j)}{P_{R}(i) P_{F}(j)}
$$

where $P_{R F}$ is the normalized joint gray level histogram of images $R$ and $F, P_{R}$ and $P_{F}$ are the normalized marginal histograms of the two images.

Fusion factor $(F F)$ is a metric based on $M I$ aimed to integrate complementary information from multiple sources. For source images $(A$ and $B)$, the quality of fused image $(F)$ is defined by $F F$ as follows:

$$
F F=M I_{A F}+M I_{B F}
$$

Objective edge based quality $(Q E)$ metric is based on Sobel operator edge statistics and calculated as:

$$
Q E=\frac{\sum_{i=1}^{n} \sum_{j=1}^{m} k^{a}(i, j) w^{a}(i, j)+k^{b}(i, j) w^{b}(i, j)}{\sum_{i=1}^{n} \sum_{j=1}^{m} w^{a}(i, j)+w^{b}(i, j)}
$$

where $w^{a}$ ve $w^{b}$ are the edge magnitudes for two source images, $k^{a}$ ve $k^{b}$ edge preservation coefficients. For detailed explanation of this metric, see [16].

The PSNR, which expressed with regard to the logarithmic decibel scale, is the ratio between a signal's maximum possible power and the power of corrupting noise and given as follows:

$$
P S N R=10 \log _{10}\left(\frac{L^{2}}{\frac{1}{M \times N} \sum_{i=1}^{M} \sum_{j=1}^{N}(R(i, j)-F(i, j))^{2}}\right)
$$

where $L$ is the number of gray levels.

The SSIM is used for calculating the similarity between two images. SSIM is calculated on consequent windows of an image. The similarity between window $R$ and $F$ of a common window size (e.g. $8 \times 8$ ) is:

$$
\operatorname{SSIM}(R, F)=\frac{\left(2 \mu_{R} \mu_{F}+c_{1}\right)\left(2 \sigma_{R F}+c_{2}\right)}{\left(\mu_{R}^{2}+\mu_{F}^{2}+c_{1}\right)\left(\sigma_{R}^{2}+\sigma_{F}^{2}+c_{2}\right)}
$$

where; $\mu_{R}$ is the average of $R, \mu_{F}$ is the average of $F, \sigma^{2}{ }_{R}$ is the variance of $R, \sigma_{F}^{2}$ is the variance of $F, \sigma_{R F}$ is the covariance of $R$ and $F, c_{1}=\left(k_{1} L\right)^{2}$ and $c_{2}=\left(k_{2} L\right)^{2}$ are the two variables to stabilize the division, $L$ is the dynamic range (typically 255 for 8-bit gray level images), $k_{1}=0.01$ and $k_{2}=0.03$ [17]. SSIM index produces a value between -1 and 1 . 


\section{AN INTERACTIVE WEB BASED IMAGE FUSION APPLICATION}

In this section, the proposed interactive web based application is introduced. In order to develop the application two major underlying technologies: Microsoft ASP.NET and MATLAB are used. The reason of the using Microsoft ASP.NET for web interface is that it gives the easy web development skills. MATLAB is used to realize the image fusion tasks, since it presents huge libraries for image processing, wavelet, optimization, and so on.

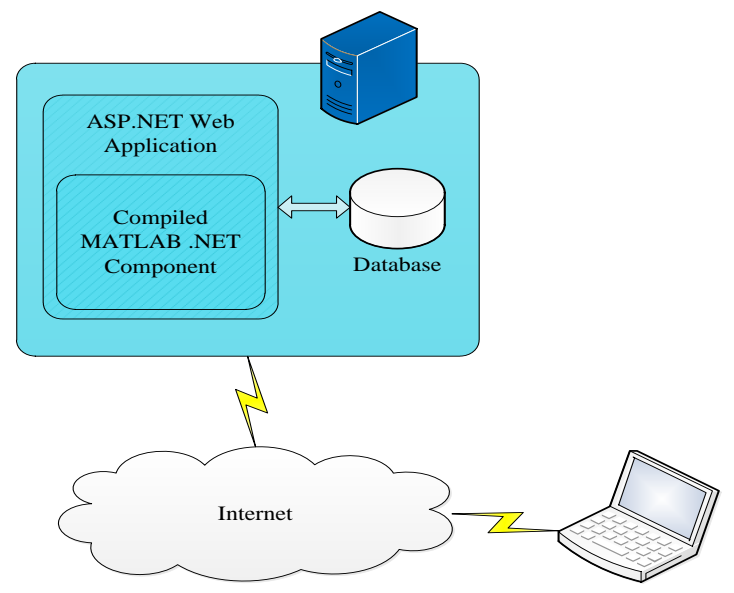

Fig. 1. Architecture of the proposed application

The main purpose of the proposed application is providing an easy to use web page that allows users to access image fusion sources and methods from any place. Users can also change several parameters of the fusion techniques to observe the effects of parameters on the fusion results. The application consists of only one page that acquire source images and the parameter values of the methods from the user and presents visual and quantitative fusion results.

The proposed application is composed of the web interface, the fusion engine and the database. The web interface has a user-friendly design which allows users to submit the images to be fused, change the parameters of the methods, and get numeric and visual fusion results within minutes. In the design of the web interface, ASP.NET technology (with AJAX extensions) is preferred in order to make the design userfriendly and increase the interactivity of the website. The fusion and evaluation stages are carried out in a compiled MATLAB .NET component which called as fusion engine. That component has implementation of various methods and quality metrics that mentioned in this paper. Fusion engine is developed in MATLAB environment and then embedded into web site. The last component of the application is a MySQL based database that used to save the fusion results. The basic architecture of the proposed application is illustrated in Fig. 1.

In Fig. 2, user data form and parameter selection sections of the main page can be seen. Users are free to decide whether or not to provide their personal information by entering it in the form. Source images need to be uploaded prior to start fusion process. Users can choose the source images from their computer as Windows Bitmap format (.BMP). Users can also reach image database for previously presented multi-focus and multi-sensor test images. According to the uploaded source images, users choose the fusion type as multi-focus or multi-sensor. If the multi-focus is selected as the fusion type, basic averaging, LP, DWT, BBIF and SDMIF methods will be available on the parameter selection section. If the type is selected as multi-sensor, basic averaging, LP, DWT and ORMSIF methods will be available. The parameter sets consist of decomposition level selection and consistency check option for LP, decomposition level and filter family choice for DWT, and block size and focus measure selection and consistency check option for BBIF method. User can select the iteration number, population number, algorithm, crossover rate and mutation rate for the SDMIF method. At last, the parameters of the ORMSIF method are iteration number, population number, algorithm, crossover rate, mutation rate, number of region, and wavelet family. For the SDMIF and ORMSIF methods if the algorithm is selected as $\mathrm{ABC}$, limit parameter will be available instead of mutation and crossover rate parameters. 
ICIT 2015 The $7^{\text {th }}$ International Conference on Information Technology

doi:10.15849/icit.2015.0076 @ ICIT 2015 (http://icit.zuj.edu.jo/ICIT15)

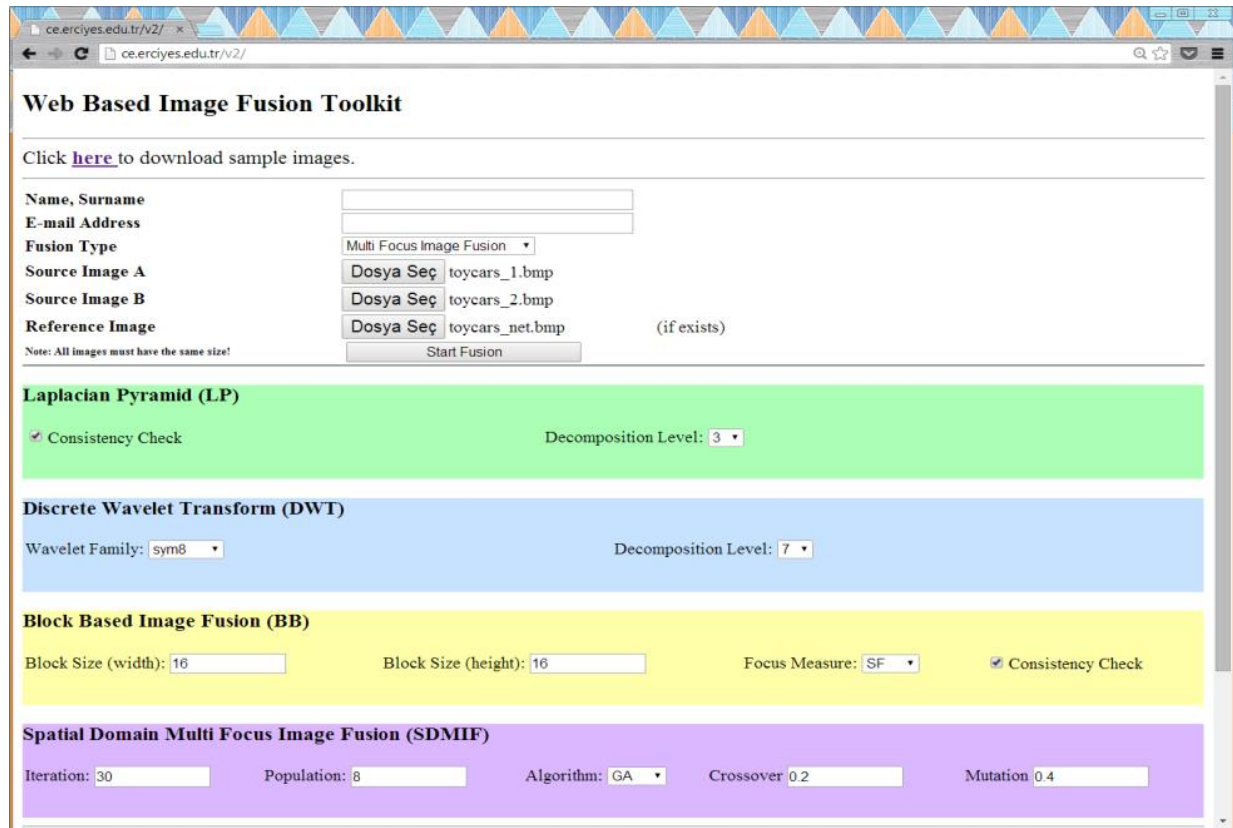

Fig. 2. Main page of web based application. (Parameter Section)

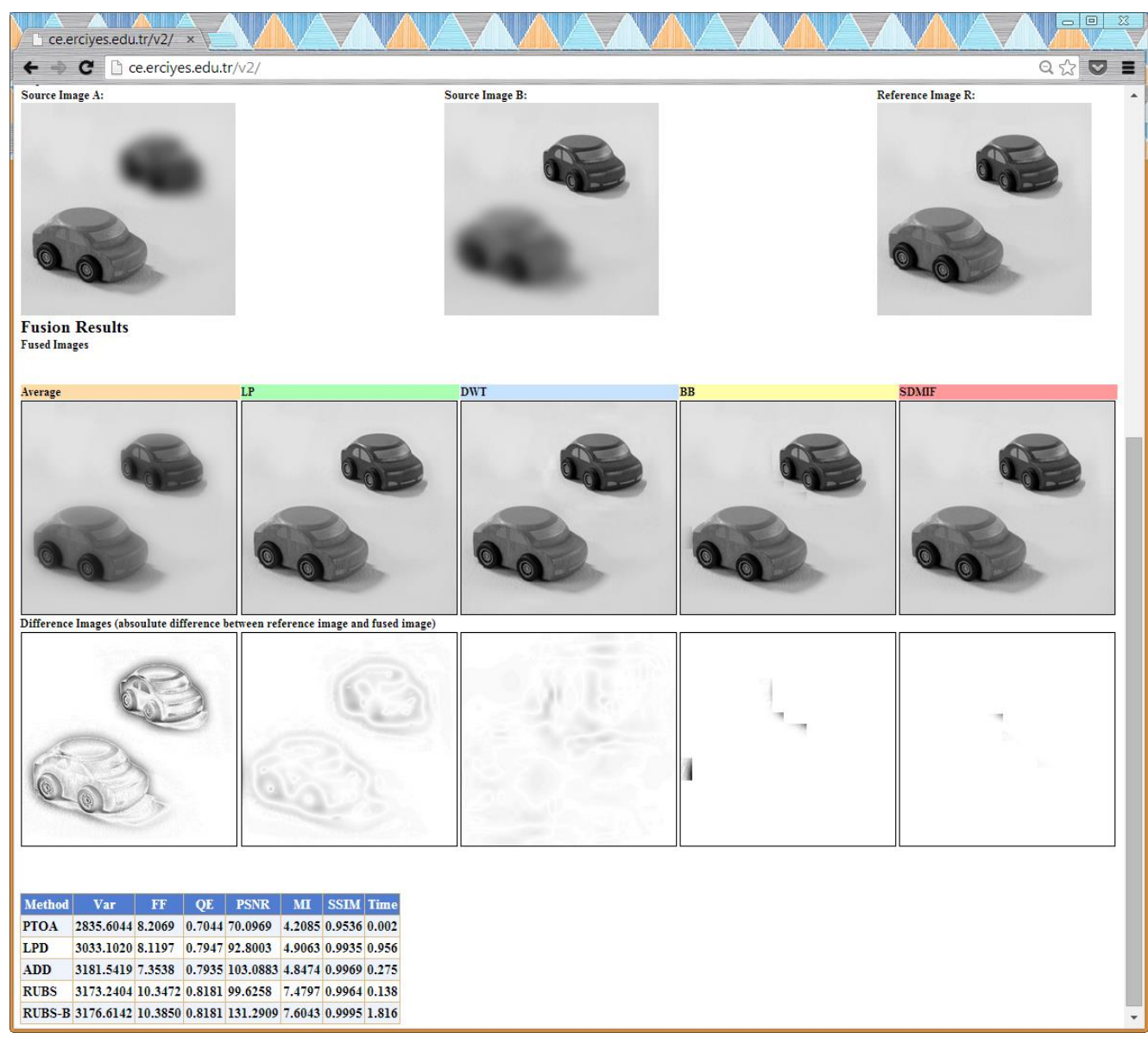

Fig. 3. Main page of web based application. (Parameter Section) 
Fig. 3 shows the visual and quantitative results sections of the main page. The source images and the reference image (if exists) that submitted by user are also shown in this section. Fused and difference image of corresponding fusion methods are illustrated in a table. In order to simplify the visual evaluation, the absolute error images which are computed by subtracting the fused image from the reference image are also shown under the fusion results. If there is no reference image, the difference error images are obtained by subtracting the fused image from the each source images.

At last, quantitative results are also are given as a table at the bottom of the web page. The table consists of both of the objective metrics and reference based metrics. Included objective metrics are VAR, FF and QE and included reference based metrics are MSE, PSNR, MI and SSIM. Reference based metric results are only available when a reference image is submitted by user.

\section{CONCLUSION}

In this paper, a web based interactive application for multi-focus and multi-sensor image fusion that includes wellknown image fusion methods is presented.

The proposed application has an easy to use and interactive web interface. It allows the users to visualize the image fusion methods, understand the basic principles of the image fusion, compare the methods by means of performance and robustness and evaluate the methods with different inputs, and parameter values. Moreover, the users can use the web based application from anywhere without installing a preliminary other software packages. The proposed application enables users to conduct experiments on different image fusion methods.

\section{REFERENCES}

[1] V. Aslantas and R. Kurban, "A comparison of criterion functions for fusion of multi-focus noisy images," Optics Communications, vol. 282, pp. 32313242, Aug 2009.

[2] V. Aslantas and A. N. Toprak, "A pixel based multifocus image fusion method," Optics Communications, vol. 332, pp. 350-358, 2014.

[3] Z. B. Wang, Y. D. Ma, and J. Gu, "Multi-focus image fusion using PCNN," Pattern Recognition, vol. 43, pp. 2003-2016, Jun 2010.

[4] V. Aslantas and E. Bendes, "Differential evolution algorithm based spatial multi-sensor image fusion," in ICINCO 2014 - Proceedings of the 11th
International Conference on Informatics in Control, Automation and Robotics, 2014, pp. 718-725.

[5] R. S. Blum, Z. Xue, and Z. Zhang, "An Overview of Image Fusion," in Multi-Sensor Image Fusion and Its Applications, R. S. Blum and Z. Liu, Eds., ed: Taylor\&Francis, 2006, pp. 1-36.

[6] V. Aslantas, R. Kurban, A. N. Toprak, and E. Bendes, "An interactive web based toolkit for multi focus image fusion," Journal of Web Engineering, vol. 14, pp. 117-135, 2015.

[7] D. Mueller, A. Maeder, and P. O'Shea, "The generalised image fusion toolkit (GIFT)," in Insight journal, special issue : MICCAI workshop on open science, 2006, pp. 1-16.

[8] O. Rockinger. Image Fusion Toolbox for Matlab. Available: http://www.metapix.de/toolbox.html

[9] G. Bebis, D. Egbert, and M. Shah, "Review of computer vision education," IEEE Transactions on Education, vol. 46, pp. 2-21, Feb 2003.

[10] R. Blum, Z. Xue, and Z. Zhang, An Overview of Image Fusion: CRC Press, 2005.

[11] G. Pajares and J. M. de la Cruz, "A wavelet-based image fusion tutorial," Pattern Recognition, vol. 37, pp. 1855-1872, Sep 2004.

[12] S. Li, J. T. Kwok, and Y. Wang, "Combination of images with diverse focuses using the spatial frequency," Information Fusion, vol. 2, pp. 169-176, 2001.

[13] V. Aslantas and R. Kurban, "Fusion of multi-focus images using differential evolution algorithm," Expert Systems with Applications, vol. 37, pp. 8861$8870,2010$.

[14] V. Aslantas, E. Bendes, R. Kurban, and A. N. Toprak, "New optimised region-based multi-scale image fusion method for thermal and visible images," IET Image Processing, vol. 8, pp. 289-299, 2014.

[15] Q. Guihong, Z. Dali, and Y. Pingfan, "Information measure for performance of image fusion," Electronics Letters, vol. 38, pp. 313-315, 2002.

[16] C. S. Xydeas and V. Petrovid, "Objective image fusion performance measure," Electronics Letters vol. 36, pp. 308-309, 2000.

[17] Z. Wang, A. C. Bovik, H. R. Sheikh, and E. P. Simoncelli, "Image quality assessment: From error visibility to structural similarity," IEEE Transactions on Image Processing, vol. 13, pp. 600-612, Apr 2004. 